\section{A retrospective analysis of the impact of toxicological diagnostics on clinical decision making in cases of acute drug poisoning}

\author{
Janne H. Liisanantti,' Suvi Lehtiniemi,' \\ Tero I. Ala-Kokko² \\ 'Division of Intensive Care Medicine, \\ Department of Anesthesiology, Oulu \\ University Hospital; ${ }^{2}$ Anesthesiology and \\ Intensive Care, Oulu University Hospital, \\ Oulu, Finland
}

\begin{abstract}
The outcome of acute drug poisoning is good. In only a few occasions specific treatments are needed. Toxicological screenings are recommended when acute drug poisoning is suspected. In this retrospective observational study the impact of routine screening on treatment decisions was analyzed. All patients with acute drug poisoning admitted to the emergency department of our university hospital during one year (2013) were retrospectively analyzed. The patients were categorized into two groups: those who received specific therapies due to the poisoning and those who received only symptomatic treatment. Results: there were a total of 318 cases with acute drug poisoning of which 120 led to intensive care treatment. Toxicological screening was performed in 225 cases (70.8\%). The screening tests were more often taken from patients who were unconscious ( $89 \%$ ) or had altered consciousness $(79 \%)$ than from patients with normal consciousness $(63 \%, \mathrm{P}<0.001)$. The rate of specific treatment was higher among screened patients compared with patients without screening (18.7 vs $1.1 \%$, $\mathrm{P}<0.001)$. However, unexpected screening results were found in 37 of the 225 screened patients out of whom only 6 cases received specific treatment. Most patients with acute drug poisoning were toxicologically screened, but every sixth had an unexpected finding. The rate of patients with unexpected screening result receiving specific treatment was low.
\end{abstract}

\section{Introduction}

Acute drug poisoning is a relatively common cause of emergency department (ED) admissions. The outcome in hospital care is good even with the cases treated in the intensive care unit (ICU) with the mor- tality rate less than $3 \%,{ }^{1-4}$ and in a Norwegian study by Lund et al the hospital mortality was even less, $0.8 \% .^{5}$ The hospital length of stay (LOS) is usually less than one day. ${ }^{1-5}$ The causes of intoxication include analgesics and psychotropic drugs, such as antidepressants and anxiolytics. ${ }^{1,5}$ In Finland the most typical cause of acute drug poisoning is the intake of psychotropic products in association with alcohol intoxication. ${ }^{1}$ Similar findings have been reported from Oslo, Norway, but for instance in a Scottish series paracetamol was the most frequent cause of poisoning. 5,6

The recommended cornerstones in the management of patients with acute drug poisoning are stabilization of the vital functions and the use of activated charcoal to prevent the absorption of the ingested agent. Toxicological screenings can be used for diagnostics and to guide the management of the patients. ${ }^{7,8}$ The diagnostics in acute poisoning is challenging as shown in one Finnish and one Norwegian study where the suspected causes of poisoning and the results of the toxicological screenings did not correlate well with patient history. ${ }^{9,10}$ However, the evidence supporting the use of toxicological screenings is lacking. There are studies questioning the use of screenings, but they are with limited number of patients or including also not poisoned patients ${ }^{11-13}$ or conducted with pediatric population. ${ }^{14}$

The outcome of the patient with acute drug poisoning is good, but the impact of the used screenings on the outcome is unclear. Therefore, we decided to retrospectively analyze the impact of the performed toxicological screenings on the decisions to start specific therapies or extracorporeal treatment.

\section{Materials and Methods}

\section{Setting and study design}

This retrospective observational study was conducted in Oulu University Hospital located in Northern Finland. Due to the retrospective study design no statement was obtained from the hospital's ethics committee. The protocol was approved by the North-Ostrobothnian hospital administration.

All the patients with acute drug poisoning admitted to the hospital ED between January 1, 2013, and December 31, 2013, were included in the study. According to the local guidelines, poisoned patient is admitted to ICU if there is a need of airway management, if there is a risk of the need of airway management, need of vasoactive drugs
Correspondence: Janne H. Liisanantti, Division of Intensive Care Medicine, Department of Anesthesiology, Oulu University Hospital, 90029 Oulu, Finland.

E-mail: janne.liisanantti@ppshp.fi

Key words: Acute drug poisoning; Extracorporeal treatment; Diagnostics; Toxicological screening.

Contributions: Janne Liisanantti, Suvi Lehtiniemi and Tero Ala-Kokko equally contributed in design of the study, data analysis, and writing of the manuscript. Suvi Lehtiniemi and Janne Liisanantti retrieved the data from the medical records

Conflict of interest: the authors declare no potential conflict of interest.

Received for publication: 22 September 2016. Revision received: 23 March 2017.

Accepted for publication: 24 March 2017.

This work is licensed under a Creative Commons Attribution 4.0 License (by-nc 4.0).

(C) Copyright J.H. Liisanantti et al., 2017

Licensee PAGEPress, Italy

Emergency Care Journal 2017; 13:6302

doi:10.4081/ecj.2017.6302

or need of specific extracorporeal treatment for acute poisoning. In other cases patients are admitted to normal wards. The patients were identified from the hospital's discharge registry using ICD-10 diagnosis codes T36-T50.9. The patient data were manually retrieved from the medical records and hospital's laboratory system. The data consisted of patient demographics, including hospital LOS; complications, such as pneumonia, seizures, rhabdomyolysis and liver failure; and hospital mortality. The cause of poisoning was determined using the screening results, and if these were lacking, the most likely cause was recorded on the basis of the patient's medical history. Patients were categorized as conscious when Glasgow Coma Scale (GCS) score was 14-15, as having altered level of consciousness when GCS score was 9-13, and as unconscious when GCS score was less than 9. Poisoning severity score (PSS) was determined using medical records. Poisoning was considered severe if PSS was above $2 .{ }^{15}$

The patients were divided into two groups by the need of specific therapies: i) patient received specific therapies, including $\mathrm{N}$-acetyl cysteine (NAC) infusion, or forced alkaline diuresis; or the patient received extracorporeal treatment, including acute hemodialysis, acute hemodiafiltration, hemoperfusion, or molecular adsor- 
bent recycling system (MARS); ii) patient received symptomatic therapy only, including management of vital functions. Use of activated charcoal was included in symptomatic therapy.

\section{Toxicological diagnostics}

Hospital laboratory provides an immune chemical toxicological screening set for urine amphetamine, methamphetamine, benzodiazepines, cannabinoids, cocaine, opioids, and buprenorphine. Paracetamol poisoning is screened using homogenous enzyme-immunoassay method that produces a measurement of blood paracetamol concentrations. Siemens Advia Chemisty XPT -device is used for the analyses (Siemens Healthcare GmbH, Erlangen, Germany). There are no standard guidelines for toxicological screening in the unit.

\section{Statistical analysis}

The statistical analysis was performed using IBM SPSS Statistics22 software. Continuous variables are expressed as medians with 25th and 75th percentiles unless otherwise stated, and proportional data are expressed using total number (n) and percentage (\%). Differences were considered to be statistically significant with $\mathrm{P}$ value less than 0.05 . The statistical significance of proportional data was tested using Pearson chi-square, and medians between the groups were tested using nonparametric Mann-Whitney test.

\section{Results}

There were a total of 32,089 admissions that led to hospitalization in the ED during the study period, and in 318 of these admissions $(1.0 \%)$ the patient had the discharge diagnosis of acute drug poisoning and was included in the study. Of these 318 patients, $169(53.1 \%)$ were males, and the median age of the study population was 33.0 years (25.8-47.0). A total of 120 hospital admissions $(37.7 \%)$ led to an ICU admission. The median hospital LOS was 1 day (1-2) (Table 1). The toxicological screening from urine was taken in 202 cases (63.5\%) and blood paracetamol concentration was screened in $225(70.8 \%)$ cases. Urine samples were also screened from all patients screened for paracetamol; thus, the total number of screened patients was 225 , which was $70.8 \%$ of all patients admitted to the ED due to acute drug poisoning. The screening tests were more often taken from unconscious patients (46 of $52,88.5 \%$ ) or patients with altered consciousness (67 of $85,78.8 \%$ ) compared with patients who had a normal level of consciousness (114 of 180, 63.3\% $\mathrm{P}<0.001)$. The screened patients were more often admitted to the ICU than the patients who were not screened (106 of $225,47.1 \%$ vs 14 of $93,15.1 \%, \mathrm{P}<0.001$ ) (Table 2).

The specific therapies or specific extracorporeal treatment was required in 42 $(17.5 \%)$ of the screened 225 cases. The most common required specific therapy was NAC infusion, which was used in 31 admissions (9.7\% of all the 318 admissions). Forced alkaline diuresis was used 9 times ( $2.8 \%$ of all 318 admissions). Hemodialysis was used 4 times (1.3\% of all 318 admissions), hemodiafiltration was used twice, and one patient received MARS therapy. Patients requiring special therapies or specific extracorporeal treatment had a higher rate of paracetamol ingestions than patients who received symptomatic therapy only, (31 of 42 [73.8\%] vs 4 of 183 [2.2\%], $\mathrm{P}<0.0001)$, and they also had longer median ICU stay (28 hours vs 12 hours, $\mathrm{P}<0.0001)$ and longer median hospital stay (1.5 days $v s$ 1 day, $\mathrm{P}=0.001)$. Patients with symptomatic

Table 1. Demographics of the 318 patients admitted to hospital due to acute drug poisoning.

\begin{tabular}{|c|c|c|c|c|}
\hline & $\begin{array}{l}\text { Total } \\
\mathrm{N}=318\end{array}$ & $\begin{array}{l}\text { Patients without } \\
\text { toxicological screening }(\mathrm{N}=93)\end{array}$ & $\begin{array}{l}\text { Patients with } \\
\text { toxicological screening ( } N=225)\end{array}$ & $\mathbf{P}$ \\
\hline Gender f/m & $149 / 169$ & $46 / 47$ & $122 / 103$ & 0.549 \\
\hline Age, median, y & $33.0[25.8-47.0]$ & $36.0[26-52]$ & $32[26-46]$ & 0.166 \\
\hline Suicide or DSH & 239 & $72[79.1]$ & $167[75.2]$ & 0.461 \\
\hline $\begin{array}{l}\text { Level of consciousness at ED } \\
\text { Unconscious; GCS <9 } \\
\text { Altered level of consciousness; GCS 9-13 } \\
\text { Conscious; GSC 14-15 }\end{array}$ & $\begin{array}{l}52(16.3) \\
85(26.8) \\
180(56.6)\end{array}$ & $\begin{array}{c}6(6.5) \\
17(18.3) \\
70(75.3)\end{array}$ & $\begin{array}{c}46(20.5) \\
68(30.4) \\
110(49.1)\end{array}$ & $<0.001$ \\
\hline $\begin{array}{l}\text { Cause of the poisoning } \\
\text { Atypic neuroleptics } \\
\text { Benzodiazepines } \\
\text { Paracetamole } \\
\text { Antiepileptics } \\
\text { SSRI } \\
\text { Opioids } \\
\text { Neuroleptics } \\
\text { TAD } \\
\text { Other }\end{array}$ & $\begin{array}{c}54(17.0) \\
43(13.5) \\
35(11.0) \\
27(8.5) \\
21(6.6) \\
21(6.6) \\
15(4.7) \\
11(3.5) \\
91(28.6)\end{array}$ & $\begin{array}{c}22(23.7) \\
12(12.9) \\
0 \\
7(7.5) \\
5(5.4) \\
7(35.5) \\
5(5.4) \\
2(2.2) \\
33(35.5)\end{array}$ & $\begin{array}{c}32(14.2) \\
31(13.8) \\
35(15.6) \\
20(8.9) \\
16(7.1) \\
14(6.2) \\
10(4.4) \\
9(4.0) \\
58(25.8)\end{array}$ & 0.006 \\
\hline Multiple ingestion & $252(79.2)$ & $66(71.0)$ & $186(82.7)$ & 0.270 \\
\hline Specific therapy or extracorporeal treatment & $43(13.5)$ & $1^{*}(1.1)$ & $42(18.7)$ & \\
\hline Intubation & $45(14.2)$ & $5(5.4)$ & $40(17.8)$ & 0.064 \\
\hline $\mathrm{PSS}>2$ & $20(6.3)$ & $5(5.4)$ & $15(16.7)$ & 0.660 \\
\hline Hospital LOS, median, d & $1(1-2]$ & $1(1-2)$ & $1(1-2)$ & 0.270 \\
\hline ICU admission & $120(37.7)$ & $14(15.1)$ & $106(47.1)$ & $<0.001$ \\
\hline ICU LOS, median, h & $14(8-20)$ & $15(6-17)$ & $13(9-20)$ & 0.889 \\
\hline Complication & $25(7.9)$ & $4(4.3)$ & $21(9.3)$ & 0.129 \\
\hline
\end{tabular}

ED, emergency department; GCS, Glascow Coma Scale; ICU, intensive care unit; LOS, length of stay; PSS, poisoning severity score; TAD, tricyclic antidepressants *One patient received NAC for indication not paracetamol poisoning 
therapy were more often unconscious at ED [101 of $183(55.5 \%)$ vs 13 of $42(31.0 \%)$, $\mathrm{P}=0.004]$. There were no differences in unexpected screening results, the rate of complications, or admissions to ICU between the groups.

Unexpected findings were recorded in $37(16.4 \%)$ of the screened 225 patients, and $6(2.7 \%)$ of them were among those 42 patients who required specific therapies or specific extracorporeal treatment. Three of these were paracetamol poisonings recognized by the screening. The other three were identified using specific laboratory tests due to clinical suspicion (Table 3 ).

In 178 cases $(78.0 \%)$ the results of the performed screenings or diagnostic tests were commented in the medical records made in the ED.

All patients had a good hospital outcome. Complications were recorded in 25 patients $(7.8 \%)$, including 9 cases of seizures $(2.8 \%), 8$ cases of pneumonia $(2.5 \%), 5$ patients who had a prolonged stay in the hospital due to altered consciousness,
2 patients who developed a liver failure, and 1 patient with rhabdomyolysis. The recorded complications were not related to the toxicological screenings used; there were 4 (4.4\%) recorded complications among nonscreened patients and $21(9.2 \%)$ recorded complications among screened patients $(\mathrm{P}=0.148)$

\section{Discussion}

The main finding of the present study was that $70 \%$ of the admissions due to poisoning involved screening but only onefifth of the screened patients received specific care or extracorporeal treatment. Moreover, the rate of unexpected screening results leading to specific care or extracorporeal treatment was less than 3\% (6 of 225).

We evaluated a non-selected patient population in a university hospital setting aiming to study the usefulness of toxicolog- ical screenings to detect the need of specific care or extracorporeal treatments. The previous studies focusing the use of screenings have questioned the impact of toxicological screenings in poisoned patients. ${ }^{11-14}$ However, in these papers the patients' characteristics differenced from the present study including lower number of unconscious patients and other than poisoned patients. ${ }^{11-13}$

In the present study, the patient demographics, causes of admissions, rate of ICU admissions, and the outcome are comparable with the Norwegian study by Lund et al published in $2012 .{ }^{5}$ Corresponding with this Norwegian study, the need for specific therapies or extracorporeal treatment was low also in our material. We did not find differences in outcome between the patients who required specific therapies or extracorporeal treatments and those who needed symptomatic therapy, but the median hospital LOS and ICU LOS among patients with symptomatic care were shorter. Also, the rate of unexpected screening results was equal

Table 2. The 225 toxicologically screened (toxicological urine samples or paracetamol screening) drug poisoned patients divided into the two groups; need of specific therapies of extracorporeal treatment ( $\mathrm{N}$-acetyl cysteine or forced alkaline diuresis, acute hemodialysis, hemodiafiltration, or molecular adsorbent recirculating system) or symptomatic care.

\begin{tabular}{|c|c|c|c|}
\hline & $\begin{array}{l}\text { Specific therapies or extracorporeal treatment } \\
\qquad \mathrm{N}=42(17.5)\end{array}$ & $\begin{array}{l}\text { Symptomatic therapy } \\
\qquad \mathrm{N}=183(81.3)\end{array}$ & P \\
\hline Age, median, y & 30 [21-52] & $32[26-46]$ & 0.774 \\
\hline Gender $\mathrm{f} / \mathrm{m}$ & $22 / 18$ & $77 / 104$ & 0.101 \\
\hline $\begin{array}{l}\text { Unexpected finding in the screening } \\
\text { or in the specific laboratory test }\end{array}$ & $6(14.3)$ & $31(16.9)$ & 0.676 \\
\hline Unconscious at ED & $13(31.0)$ & $101(55.5)$ & 0.004 \\
\hline Suicide and DSH & $32(78.0)$ & $135(74.6)$ & 0.643 \\
\hline $\begin{array}{l}\text { Specific therapies } \\
\text { NAC } \\
\text { Hemodialysis } \\
\text { Hemodiafiltration } \\
\text { Forced alcaline diuresis } \\
\text { MARS }\end{array}$ & $\begin{array}{c}31(76.9) \\
4(10.0) \\
2(5.0) \\
9(22.5) \\
1(2.5) \\
\end{array}$ & & \\
\hline $\begin{array}{l}\text { Cause of the poisoning } \\
\text { Paracetamol } \\
\text { Atypic neuroleptics } \\
\text { Benzodiazepines } \\
\text { Antiepileptics } \\
\text { SSRI } \\
\text { Opioids } \\
\text { Neuroleptics } \\
\text { TAD } \\
\text { Other }\end{array}$ & $\begin{array}{c}31(73.8) \\
0 \\
0 \\
1(2.5) \\
0 \\
0 \\
1(2.4) \\
2(4.8) \\
7(16.7)\end{array}$ & $\begin{array}{c}4(2.2) \\
32(17.5) \\
31(16.9) \\
19(10.4) \\
16(8.7) \\
14(7.7) \\
9(4.9) \\
7(3.8) \\
51(27.9)\end{array}$ & $<0.0001$ \\
\hline Multiple ingestion & $32(76.2)$ & $154(84.2)$ & 0.219 \\
\hline Intubation & $4(9.1)$ & $41(14.9)$ & 0.242 \\
\hline $\mathrm{PSS}>2$ & $4(9.5)$ & $16(5.8)$ & 0.358 \\
\hline ICU admission & $20(47.6)$ & $86(47.0)$ & $>0.9$ \\
\hline ICU LOS, median, h & 28 [15.5-45.5] & 12 [8-18] & $<0.0001$ \\
\hline Hospital LOS, median, d & $1.5[1-3]$ & $1[1-2]$ & 0.001 \\
\hline Complication & $6(14.3)$ & $15(8.2)$ & 0.221 \\
\hline
\end{tabular}

DSH, deliberate self-harm; ED, emergency department; ICU, intensive care unit; LOS, length of stay; MARS, molecular adsorbent recirculating system; NAC, N-acetyl cysteine; PSS, poisoning severity score; SSRI, selective serotonin reuptake inhibitors; TAD, tricyclic antidepressants; PSS, poisoning severity score. 
between patients who required specific therapies or extracorporeal treatment and those with symptomatic care despite the differences in agents ingested between the groups. This result is interesting when compared to the results of Pohjola-Sintonen at al and Heyerdahl et al, which showed that there were high discrepancies between clinical and laboratory assessments most often with products affecting central nervous system. ${ }^{9,10}$ These discrepancies were considered clinically important in $11 \%$ to $18 \%$ of the cases. In our series $16 \%$ of the screening results were considered unexpected. However, in our study the rate of unexpected screening results leading to specific treatment was less than three percent. Also in the previous Finnish series by PohjolaSintonen et al the discrepancies that were considered clinically significant did not lead to specific therapies and were not related to recorded complications. ${ }^{9}$ In the present results it is notable that only 3 of the 6 unexpected findings among patients who required specific therapies were detected by the routine screening (paracetamol) and in only 2 of these cases the patient had a lowered level of consciousness. The detection of the other 3 cases required specific laboratory testing (lithium and salicylate concentration). In the light of the present results the use of toxicological screening without suspicion of poisoning requiring specific therapies or extracorporeal treatment can be questioned. In most cases of the ICU admitted patients the screening did not lead to in any specific treatment. The decision to admit the patient to ICU was more related to vital functions than the screening results. Furthermore, the present study population included only $6 \%$ of severe poisonings according to PSS and thus the role of toxicological investigations could be questionable. The history of substance abuse of hos- pitalized patients can be unreliable. In a study conducted in Tennessee, United States, a total of 1500 ED patients were interviewed, and the interviews were compared to toxicological screening results. This comparison revealed discrepancies in self-reported and screened abuse of opioids, benzodiazepines, cannabis and stimulants. However, it is notable that the patients included in the study had not been admitted to hospital for poisoning-related reasons. ${ }^{16}$ According to the present results, instead of the diagnostics of the known or suspected drug-poisoned patients, the screenings could be used in differential diagnostics of altered level of consciousness when other severe causes such as neurological diseases are ruled out. This way the screening may have an impact on care but that can also be debated. ${ }^{12}$ In most cases the poisoning is a self-correcting condition, in which the body requires time to metabolize and eliminate the agent. ${ }^{5}$ In the present study, the screenings were more often used in patients with altered level of consciousness. This can lead to bias in the results since specific treatments also less often needed among these patients. It would have been interesting to see the impact of screenings on the patients management if all the patients would have been screened. However, the aim of the present study was to also to evaluate the use of the screenings, not only the impact of their use.

The outcome in the present study was good and thus similar to the outcomes reported previously. 2,5 There are recommendations that support the use of toxicological screenings for diagnostics in the patients admitted to hospital due to acute drug poisoning or in cases of suspected acute drug poisoning. ${ }^{7,8} \mathrm{We}$ were not able to show any major differences in the outcome or the rate of complications between the screened and non-screened patients. The screened patients more often presented altered level of consciousness and were admitted to the ICU, but on the other hand, two-thirds of the patients who required specific care were not unconscious at ED while the corresponding rate among those who needed symptomatic care was less than half. Considering the very low mortality and morbidity among hospitalized patients with drug poisoning, it may be asked how the outcomes of these patients may be further enhanced. The screenings would probably have had an impact on the care in every fifth screened patient $(n=42)$ if the specific therapies or specific extracorporeal treatment had been conducted according to screening results. If only taking into account patients with unexpected findings, only every seventieth screened patient $(n=3)$ benefitted from the screening, which is a very small number of patients. With limited number of patients receiving specific care as well as limited number of patients with severe poisonings the collaboration with clinical toxicologists is important when producing guidelines concerning the use of screenings to implement the right diagnostic tests.

The benefit that is possible to gain using screenings seems to be minor in this patient group with good outcome, limited number of specific treatment procedures, and relatively simple, symptomatic care. Similarly to our study patients, most of the patients in the Norwegian cohort did not receive any other treatment than supportive therapy; a minority received activated charcoal and special procedures to enhance the elimination of the harmful agent, and antidotes were used in also less than $50 \%$ of the patients..$^{5}$ The focus should be in prevention of complications and factors associated with prolonged ICU stay. Our previous study showed that prolonged ICU stays

Table 3. Patients $(n=6)$ who received special therapies or extracorporeal treatment with unexpected screening or laboratory test results.

\begin{tabular}{|c|c|c|c|c|c|c|c|c|}
\hline $\begin{array}{l}\text { Gender, } \\
\text { age }\end{array}$ & $\begin{array}{l}\text { Agent } \\
\text { ingested }\end{array}$ & $\begin{array}{l}\text { Consciousness } \\
\text { at ED }\end{array}$ & $\begin{array}{l}\text { Intake } \\
\text { motivation }\end{array}$ & $\begin{array}{l}\text { Laboratory } \\
\text { test }\end{array}$ & $\begin{array}{c}\text { ICU } \\
\text { LOS } \\
\text { (hours) }\end{array}$ & $\begin{array}{l}\text { Hospital } \\
\text { LOS } \\
\text { (days) }\end{array}$ & $\begin{array}{c}\text { Specific } \\
\text { therapy/ } \\
\text { extracorporeal } \\
\text { treatment }\end{array}$ & $\begin{array}{l}\text { Poisoning } \\
\text { severity } \\
\text { score }\end{array}$ \\
\hline $\mathrm{f}, 66$ & Lithium & Conscious & Accident & $\begin{array}{c}\text { Blood } \\
\text { lithium concentration }\end{array}$ & 120 & 6 & Hemodialysis & 2 \\
\hline $\mathrm{f}, 52$ & Paracetamol & $\begin{array}{c}\text { Altered level } \\
\text { of consciousness }\end{array}$ & Suicide & Paracetamol screening & 28 & 4 & NAC & 2 \\
\hline $\mathrm{f}, 69$ & Salicylate & Conscious & - & $\begin{array}{l}\text { Blood salicylate } \\
\text { concentration }\end{array}$ & 45 & 7 & $\begin{array}{l}\text { Hemodialysis, forced } \\
\text { alkaline diuresis }\end{array}$ & 2 \\
\hline f, 46 & $\begin{array}{l}\text { Lithium, multiple } \\
\text { ingestion }\end{array}$ & Unconscious & Suicide & $\begin{array}{l}\text { Blood lithium } \\
\text { concentration }\end{array}$ & 46 & 3 & Forced alkaline diuresis & 3 \\
\hline $\mathrm{f}, 76$ & Paracetamol & Conscious & Suicide & Paracetamol screening & - & 1 & NAC & 1 \\
\hline $\mathrm{f}, 20$ & Paracetamol & Unconscious & Recreational use & Paracetamol screening & 8 & 3 & NAC & 2 \\
\hline
\end{tabular}

ED, emergency department; ICU, intensive care unit; LOS, length of stay; NAC, N-acetyl cysteine. 
were associated with organ dysfunctions, such as hypotension and respiratory failure. ${ }^{2}$ The screenings may be useful in cases of potentially dangerous poisonings, such as lithium or paracetamol poisonings with specific treatments available, but according to our results, only with clinical suspicion. However, the present data as a single center, retrospective data should be considered as hypothesis generating. The findings call for a large prospective study to examine the impact of toxicological screenings on the treatment decisions, outcome and whether these tests could reduce the number of ICU admissions in poisoned patients without need of organ support, including airway management. We presented data of 318 patients with acute poisoning who were admitted to university hospital ED within one year. According to our knowledge this was the first study analyzing the impact of toxicological screening on the management of patients with acute drug poisoning. We included all drug-poisoned patients, mimicking the actual clinical situation. The present results are comparable with the Norwegian study in terms of patients' characteristics, including the level of consciousness on admission, need of ICU admission, and outcome, which promotes the generalizability of the present results at least to Nordic countries. ${ }^{5}$ There are limitations in this study. First, the design was retrospective. However, with this setting we were able to show afterwards the decisions that were made using the screenings. Also, the number of patients was limited, but we covered one year's admissions to a university-level hospital. A study of the impact of screenings on mortality would have required inclusion of several hundred patients, which would have expanded the time span too much. The aim of the present study was not to study the impact of screenings on outcome but the impact of the screenings on the treatment decisions. We were not able to analyze the use of screening in differential diagnostics in the patient population that showed an altered level of consciousness at the ED.

\section{Conclusions}

In conclusion, most patients with acute drug poisoning were toxicologically screened, but every sixth had an unexpected finding. The rate of patients with unexpected screening result receiving specific treatment was low.

\section{References}

1. Lapatto-Reiniluoto O, Kivisto KT, Pohjola-Sintonen $\mathrm{S}$, et al. A prospective study of acute poisonings in Finnish hospital patients. Hum Exp Toxicol 1998; 17:307-11.

2. Liisanantti JH, Ohtonen P, Kiviniemi O, et al. Risk factors for prolonged intensive care unit stay and hospital mortality in acute drug-poisoned patients: an evaluation of the physiologic and laboratory parameters on admission. J Crit Care 2011;26:160-5.

3. Lam SM, Lau AC, Yan WW. Over 8 years experience on severe acute poisoning requiring intensive care in Hong Kong, China. Hum Exp Toxicol 2010;29:757-65.

4. Heyerdahl F, Bjornas MA, Hovda KE, et al. Acute poisonings treated in hospitals in Oslo: a one-year prospective study (II): clinical outcome. Clin Toxicol 2008;46:42-9.

5. Lund C, Drottning P, Stiksrud B, et al. A one-year observational study of all hospitalized acute poisonings in Oslo: complications, treatment and sequelae. Scand J Trauma Resusc Emerg Med 2012;20:49.

6. Teo AI, Cooper JG. The epidemiology and management of adult poisonings admitted to the short-stay ward of a large Scottish emergency department. Scott Med J 2013;58:149-53.

7. Levine MB, Daniel E, Truitt CA, et al. Toxicology in the ICU: Part 1: general overview and approach to treatment. Chest 2011;140:795-806.
8. Mokhlesi B, Leiken JB, Murray P, Corbridge TC. Adult toxicology in critical care: part I: general approach to the intoxicated patient. Chest 2003;123:577-92.

9. Pohjola-Sintonen S, Kivisto KT, Vuori E, et al. Identification of drugs ingested in acute poisoning: correlation of patient history with drug analyses. Ther Drug Monit 2000;22:749-52.

10. Heyerdahl F, Hovda KE, Bjornaas MA, et al. Clinical assessment compared to laboratory screening in acutely poisoned patients. Hum Exp Toxicol 2008;27:73-9.

11. Montague RE, Grace RF, Lewis JH, Shenfield GM. Urine drug screens in overdose patients do not contribute to immediate clinical management. Ther Drug Monit 2001;23:47-50.

12. Murnion BP, Granot R, Day RO. Utility of urine drug screening: a clinical audit. Emerg Med Australas 2007;19:246-52.

13. Vakkalanka P, Rushton WF, Hardison LS, Bishop MC, Haverstick DM, Holstege CP. Evaluation of the initiation of urine drug screens intended for use in transfer patients. Am J Emerg Med 2014 Sep;32(9):1037-1040.

14. Wang GS, Deakyne S, Bajaj L, et al. The limited utility of screening laboratory tests and electrocardiograms in the management of unintentional asymptomatic pediatric ingestions. J Emerg Med 2013;45:34-38.

15. Persson HE, Sjoberg GK, Haines JA, Pronczuk de Garbino J. Poisoning severity score. Grading of acute poisoning. J Toxicol Clin Toxicol 1998; 36:205-13.

16. Rockett IRH, Putnam SL, Jia H, Smith GS. Declared and undeclared substance use among emergency department patients: a population-based study. Addiction 2006;101:706-12. 